Please quote as: Peters, C. \& Leimeister, J. M. (2019): Cloud, Hybrid Intelligence und digitale Arbeit für das Engineering von soziotechnischen Systemen. In: Boes, A. \& Langes, B. (Eds.), Die Cloud und der digitale Umbruch in Wirtschaft und Arbeit. Strategien, Best Practices und Gestaltungsimpulse. (pp. 91-99). Freiburg: Haufe Group. 


\section{Cloud, Hybrid Intelligence und Digitale Arbeit für das Engineering von soziotechnischen Systemen}

\section{Einführung}

Als Wirtschaftsinformatiker streben wir danach, Lösungen für relevante Probleme zu gestalten, die technisch stabil, ökonomisch sinnvoll und gesellschaftlich wünschenswert sind. Dies tun wir, indem wir eine soziotechnische Systemperspektive einnehmen. Dieser Artikel präsentiert dabei eine inhaltliche Leitorientierung, wie bereits bekannte und neu aufgekommene Phänomene und Konzepte ganzheitlich ineinandergreifen können.

Dies bedeutet insbesondere, neue Technologien zu verstehen und sie so einzusetzen, dass sie Nutzen stiften. Zwei Kern-Technologien, die erhebliches Disruptionspotenzial besitzen, nimmt dieser Artikel konkret in den Fokus: Cloud und Hybrid Intelligence. Diese Technologien bedürfen aber sowohl der Anwendung einer soziotechnischen System-Perspektive wie auch entsprechender Gestaltung, um die Potenziale zu heben und erfolgreiche Dienstleistungen und Geschäftsmodelle zu realisieren.

Daher zeigen wir zum einen auf, was unter Cloud (Computing/Services), Hybrid Intelligence und Crowdwork als einer Spielart von digitaler Arbeit zu verstehen ist. Zum anderen wird deren Zusammenhang aufgezeigt.

Anschließend wird auf das Engineering soziotechnischer Systeme und entsprechender Dienstleistungssysteme eingegangen, das auf Basis der zuvor vorgestellten Technologien und neuen Arbeitsformen möglich und nötig wird. Unter Engineering verstehen wir hierbei die systematische Gestaltung von Dienstleistungen 
und Dienstleistungssystemen unter Verwendung von Methoden, Modellen und Werkzeugen.

\section{Disruptive Entwicklungen: Cloud, Hybrid Intelli- gence und Digitale Arbeit}

Derzeit kann man in unterschiedlichen Bereichen disruptive Umbrüche beobachten. Cloud verändert nicht nur die Art der IT-Service-Bereitstellung, sondern bietet die Grundlage für skalierbares IT-getriebenes Geschäft. Künstliche Intelligenz und Machine Learning bieten komplett neue Anwendungsfelder, der Mix aus Künstlicher Intelligenz und menschlicher Kompetenz manifestiert sich in HybridIntelligence-Ansätzen. Und die Art, wie wir arbeiten, verändert sich stark hin zu mehr agilem und digitalem Arbeiten. Diese Entwicklungen und ihre zugrundeliegenden Konzepte sind Kern dieses Kapitels.

\subsection{Cloud}

Cloud ist sowohl wichtige Evolutionsstufe in der IT als auch das dominierende Geschäftsmodell, um IT-Infrastruktur, -Komponenten und -Applikationen anzubieten (Benlian et al. 2018). Diesbezüglich spricht man von der typischen Dreiteilung aus Infrastructure-as-a-Service (IaaS), Platform-as-a-Service (PaaS) und Software-as-a-Service (SaaS). Die Bereitstellung von Cloud Computing erfolgt dabei global, verteilt und Service-zentriert (Benlian et al. 2009). Charakteristisch ist der on-demand-Charakter (anytime) wie auch die Tatsache, dass bedarfsgerecht abgerechnet wird („pay-per-use”) und von jeder Plattform und jedem Device Zugriff ermöglicht wird (Mell/Grance 2010). Cloud Computing und entsprechende Dienstleistungen werden bezüglich des möglichen Zugriffs typischerweise unterteilt in: private (Zugriff nur innerhalb einer Organisation), shared (Zugriff für ein Konsortium aus verbündeten Organisationen), community (Zugriff limitiert auf eine bestimmte Community und ihre Mitglieder) sowie public (offener Zugriff).

Neueste Forschungsarbeiten konsolidieren drei Kern-Mechanismen (Benlian et al. 2018), die Cloud Computing zu seinem transformativen Charakter verhelfen: 
(1) Decoupling/Entkopplung:

Entkopplung bedeutet das Los- bzw. Herauslösen einzelner Systemelemente zu individuellen Service-Bausteinen, ohne dabei die Funktionsweise anderer abhängiger Systemelemente zu stören (Cusumano 2010). Dies ist strukturelle Grundlage für die im Cloud Computing typischen bedarfsgerechten Up- und Down-Skalierungen.

(2) Platformization/Plattformisierung:

Plattformisierung beschreibt den Prozess, einen attraktiven Nukleus an Dienstleistungsbündeln an einem Ort (der Plattform) bereitzustellen, auf den ein Ökosystem aus Kunden, Nutzern und anderen Stakeholdern flexibel zugreifen und auf dem es interagieren kann (Tiwana et al. 2010).

(3) Recombination/Rekombination:

Rekombination ist der Prozess, in dem Cloud Services mit anderen Technologien, innerhalb von Plattformen sowie über Plattformen hinweg, verbunden werden, um neue Innovationspotenziale zu kreieren.

Durch diese Mechanismen entstehen neue Formen der Wertschöpfung, beispielsweise für Drittanbieter, die auf dedizierten Marktplätzen für bestimmte IaaSPlattformen Komplementärangebote wie Internet-of-Things-Anwendungen offerieren und Nutzern so eine Vielzahl an Möglichkeiten bieten. Hier werden CloudInfrastrukturen also die Basis für neues IT-getriebenes Geschäft. Andere Anbieter können auf ihnen aufbauen und in diesen neuen Strukturen mit klar definierten, nutzungsabhängigen Kosten eigene Angebote in den Bereichen IoT o.ä. offerieren. Auf PaaS-Ebene wiederum werden durch intelligent orchestrierte Schnittstellen und API-Logik auf Plattformen wie Amazon Web Services Zusatzkomponenten für Lösungen in den Bereichen Künstlicher Intelligenz und Blockchain angeboten. Transaktionsabwicklung und Support ergänzen die Rundum-Pakete der Plattformen (Hahn 2016). Alle großen Softwareanbieter nutzen die vorgestellten Mechanismen außerdem, um auf SaaS-Ebene Marktplätze für Apps anzubieten und so den Funktionsumfang der eigenen Lösung signifikant zu erweitern, in Mash-ups zu kombinieren und so zum Ökosystem auszubauen (Benlian et al. 2018; Hahn 2016). 


\subsection{Hybrid Intelligence}

Die zuvor vorgestellten Cloud-Varianten und -Mechanismen als Treiber für flexibel skalierbare IT-Services sind somit technologische Grundlage für einen der aktuellen Mega-Trends: Künstliche Intelligenz (KI) bzw. "Artificial Intelligence" (AI). Das Wissenschaftsjahr 2019 steht unter diesem Motto, die Bundesregierung hat eine umfangreiche KI-Strategie (Die Bundesregierung 2018) in die Wege geleitet und will dafür bis 2025 insgesamt drei Milliarden Euro bereitstellen (Die Bundesregierung 2018). Dieser Artikel fokussiert nicht auf die neuen Machbarkeiten, die daraus direkt entstehen. Vielmehr zeigen wir auf, dass sinnvolle KINutzung sowie neue KI-Dienstleistungen und -Geschäftsmodelle insbesondere dann vielversprechend erscheinen, wenn sie künstliche und menschliche Intelligenz kombinieren. Diese Form wird Hybrid Intelligence ( $\mathrm{HI}$ ) genannt und ist definiert als die Fähigkeit, komplexe Ziele zu erreichen, indem beide Formen menschliche und künstliche Intelligenz - gemeinsam zum Tragen kommen (Dellermann et al. 2019).

Hybrid Intelligence basiert auf drei Charakteristika (Dellermann et al. 2019):

(1) Kollektive Bearbeitung: Tätigkeiten werden in HI-Systemen kollektiv über mehrere Agenten bearbeitet. Diese Agenten können Menschen oder nicht-menschliche Akteure, beispielsweise Bots, sein.

(2) Bessere Ergebnisse: Es werden in HI-Systemen bessere Ergebnisse erzielt, als es durch alleinige Verwendung einer Form von Intelligenz möglich gewesen wäre.

(3) Kontinuierliches Lernen: Über den Zeitverlauf verbessert sich das soziotechnische System, sowohl als Gesamtsystem als auch auf Ebene seiner Einzelkomponenten. Die Leistung des HI-Systems kann analog sowohl auf Basis seiner Gesamtleistung als auch anhand der Leistungssteigerung (Learning) seiner Einzelkomponenten verstanden und gemessen werden.

Die verschiedenen Formen verschiedener Intelligenz auch im Kontext von HISystemen sind in Abbildung 1 dargestellt. 


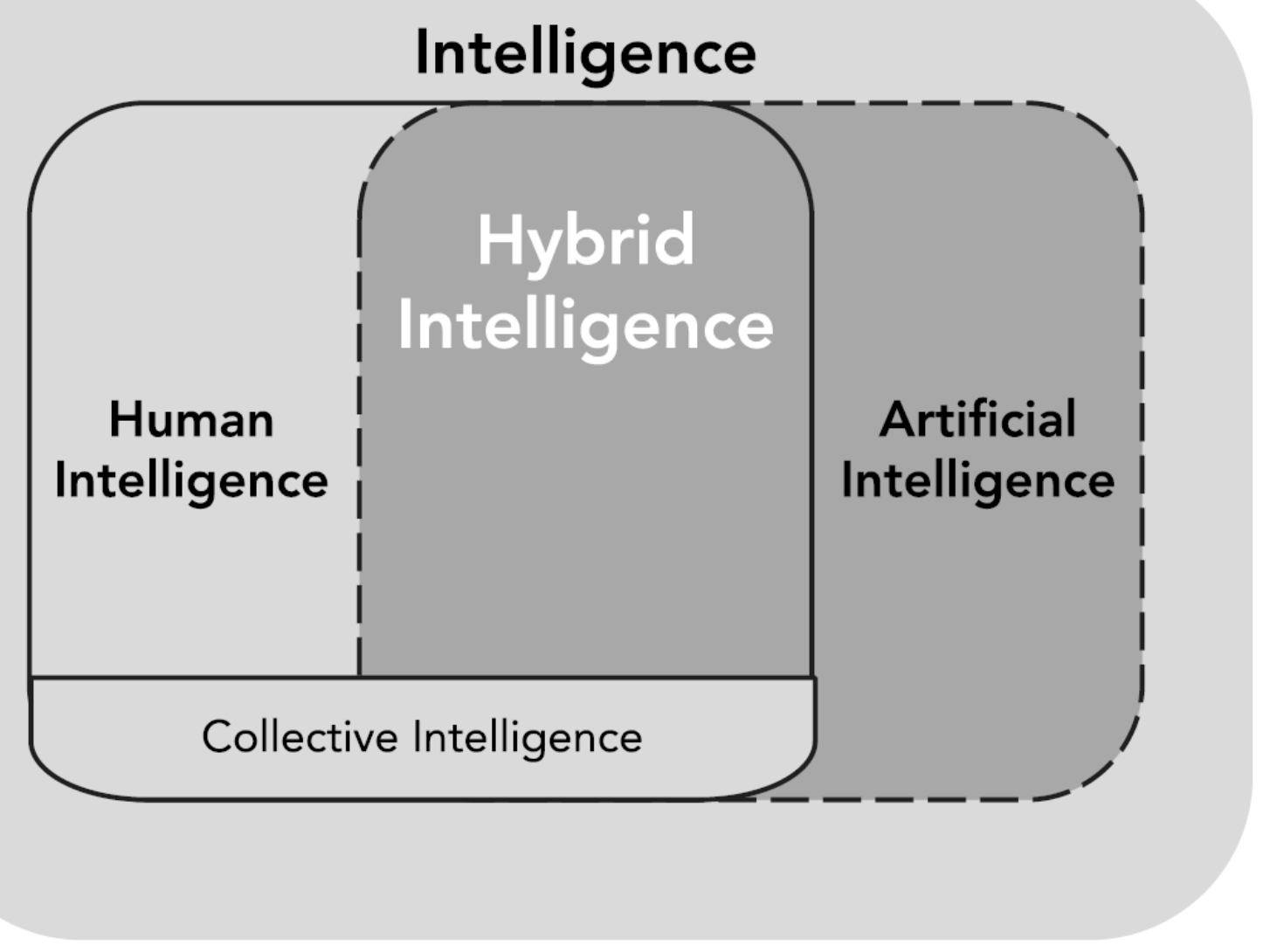

Abbildung 1: Hybrid Intelligence und andere Ausprägungen von Intelligenz, in Anlehnung an Dellermann et al. (2019)

\subsection{Digitale Arbeit}

Die Digitalisierung verändert nahezu alle Bereiche. Dies gilt auch und in besonderem Maße für die Art und Weise, wie wir arbeiten. Als eine Spielart digitaler Arbeit ist Crowdwork zu nennen. Crowdwork zeichnet sich durch vier Prinzipien aus (Durward et al. 2016):

(1) Open call: Zu erledigende Tätigkeiten werden nicht einer Person zugewiesen, sondern in einem sogenannten "open call" publik gemacht.

(2) Freiwilligkeit: Die Auswahl der Tätigkeiten erfolgt durch die Crowdworker selbst.

(3) Plattformbasiert: Die Arbeit erfolgt plattformbasiert. 
(4) Bezahlung: Im Gegensatz zu Crowdsourcing, für das die drei erstgenannten Prinzipien auch gelten, ist Crowdwork immer bezahlt.

Eine Einordnung von Crowdwork im Kontext (digitaler) Arbeit ist in Abbildung 2 veranschaulicht.

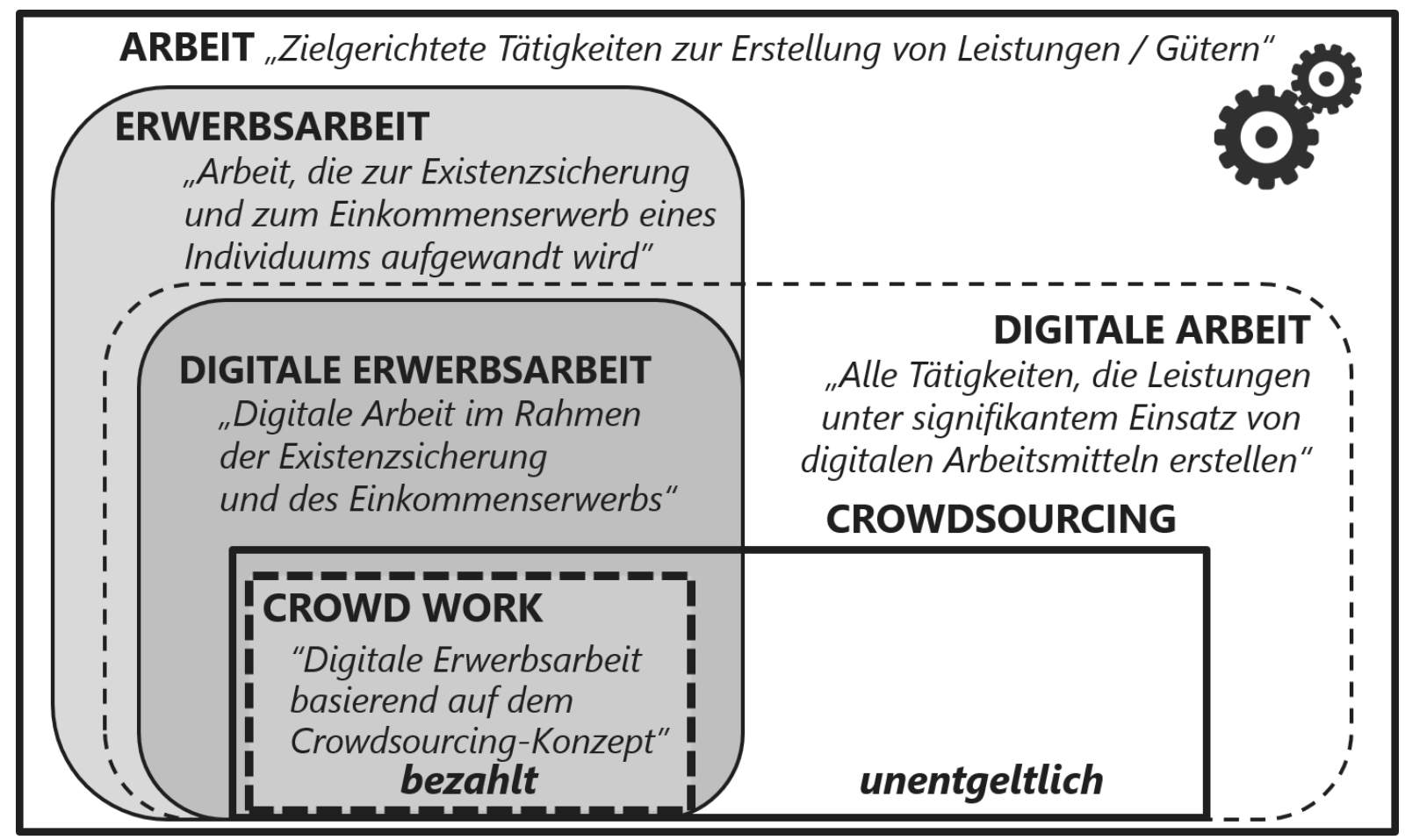

Abbildung 2: Digitale Arbeit und Crowd Work (in Anlehnung an Durward et al. 2016)

Besonders spannend wird es, wenn die neuen Konzepte, beispielsweise Crowdwork und Künstliche Intelligenz kombiniert werden. Dies führt immer dann zu attraktiven Settings, wenn menschliche Kompetenzen besonders gut auf CrowdEbene orchestriert werden können und es gleichzeitig automatisierte Bestandteile der Leistungserbringung gibt, bei denen Künstliche Intelligenz dazu beiträgt, dass selbstlernende soziotechnische Systeme bestehen. Konkret sei das Beispiel von Support-Leistungen genannt, bei denen manche Aktivitäten durch KI, manche durch die Crowd und manche auf herkömmliche Art erbracht werden können. 


\section{Zusammenhang von Cloud, Digitaler Arbeit und Hybrid Intelligence}

Während Cloud Computing und Cloud Services für die flexible, bedarfsgerechte Bereitstellung von IT-Ressourcen und -Anwendungen stehen, die insbesondere durch einen pay-per-use- und on-demand-Charakter spezifiziert werden können, gelten wie oben aufgezeigt ähnliche Prinzipien für Formen digitaler Arbeit wie Crowdwork. Hier wird beim externen Crowdwork Unternehmen die Möglichkeit gegeben, bestimmte Tätigkeiten außerhalb ihrer Unternehmensgrenzen (daher „extern") auf dedizierten Crowdworking-Plattformen bearbeiten zu lassen. Auch dies erfolgt bedarfsbezogen („on-demand"), die Crowd fungiert als "human cloud". Im Rahmen des Kapitels „Crowdworking-Plattformen als Intermediäre und Instrumente neuer Formen der Arbeitsorganisation" in diesem Buch (siehe UKS-Kapitel) gehen wir später auch auf unterschiedliche Typen dieser Plattformen ein. Im Besonderen sei in diesem Kontext auf vergleichsweise neue Entwicklungen im Bereich Crowdwork verwiesen, beispielsweise die Bearbeitung von komplexen Tätigkeiten (Mrass et al. 2018a, b) über diese Spielart digitaler Arbeit oder auch die Nutzung solcher Plattformen innerhalb von Unternehmensgrenzen („interne Crowd", Durward et al. 2019) bzw. in Mischformen, die externe und interne Crowdworking-Plattformen kombinieren („hybrid crowd", Mrass/Peters 2019).

Im Kontext des oben beschriebenen Konzepts der HI spielen sowohl Cloud als auch Crowd eine wichtige Rolle. Während die Crowd die HI-Eigenschaft der kollektiven Erbringung von Tätigkeiten mit menschlichen Agenten vorantreiben kann, sind Cloud-Services und -Infrastrukturen einer der wichtigsten Enabler, um performant und skalierbar KI- und somit HI-Lösungen in bestehende und neue Wertschöpfungsstrukturen zu integrieren. 


\section{Engineering von soziotechnischen Systemen}

Die oben beschriebenen Konzepte sowie die aufgezeigten Zusammenhänge zwischen diesen sprechen eine deutliche Sprache: Neue Technologien wie Cloud sowie KI und HI nehmen in Zeiten der Digitalisierung rasant Fahrt auf. Sie sind und werden fester Bestandteil der Systeme, mit denen und in denen wir leben und arbeiten.

Gleichzeitig sind es nicht nur die neuen technologischen Systeme und Machbarkeiten allein, die uns in Zukunft Nutzen und Wertschöpfung bringen. Vielmehr bedarf es der intelligenten und vor allem systematischen Gestaltung der soziotechnischen Systeme, um die neuen Wertschöpfungs- und Innovationspotenziale zu heben und so den Nutzen für Menschen voranzutreiben.

Dabei müssen wir nicht „bei null" anfangen, sondern können uns etablierter Instrumente zur systematischen Gestaltung von soziotechnischen Systemen bedienen, die unter Verwendung geeigneter Methoden, Modelle und Werkzeuge (Peters 2015) erfolgt und diese weiterentwickelt. Auf diese Weise lassen sich sowohl die Dienstleistungen und entsprechende Geschäftsmodelle als auch die soziotechnischen Systeme oder Dienstleistungssysteme als Ganzes „engineeren", also gestalten.

Zur systematischen Gestaltung von Dienstleistungen hat sich hier das Dienstleistungs-Engineering und -Management als Ordnungsrahmen etabliert (Leimeister 2012), das alle Lebenszyklusphasen von Dienstleistungen abdeckt. Dies ist in Abbildung 3 veranschaulicht. 


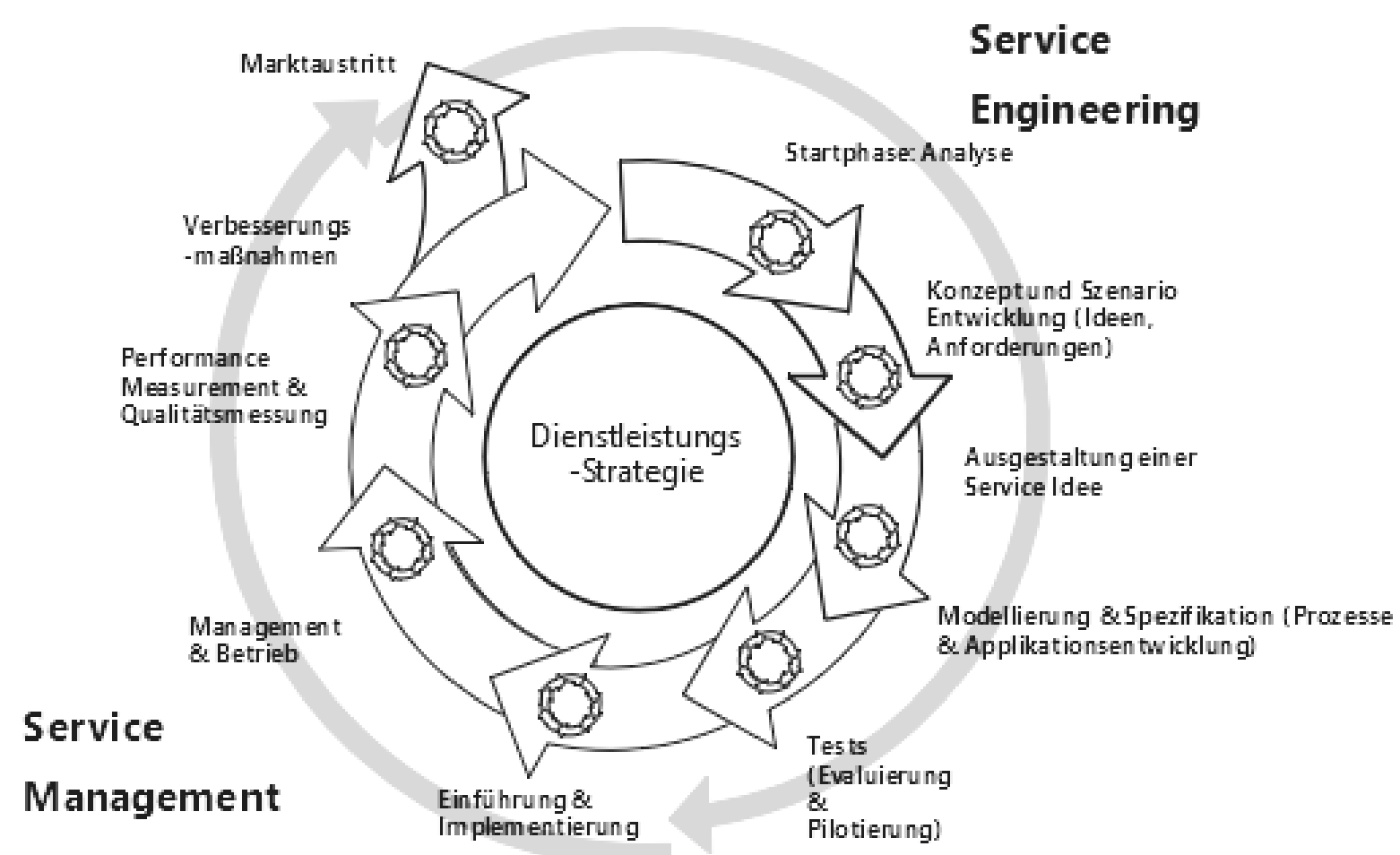

Abbildung 3: Dienstleistungs-Engineering und -Management, in Anlehnung an Leimeister (2012)

Neben der systematischen Entwicklung von Dienstleistungen gibt es auch Bestrebungen, entsprechende Geschäftsmodelle für diese Dienstleistungen integrativ systematisch mitzuentwickeln (Kleinschmidt/Peters 2017).

Bei der Gestaltung gesamter soziotechnischer Systeme wird insbesondere auf Grundlagen, Methoden, Modelle und Werkzeuge des "Service Systems Engineering" (Böhmann et al. 2014) zurückgegriffen. Hierbei sind als KernGestaltungspfade zu nennen (Böhmann et al. 2014):

(1) Engineering von Dienstleistungs-Architekturen,

(2) Engineering von Dienstleistungsinteraktionen,

(3) Engineering von Ressourcenmobilisierung innerhalb und zwischen soziotechnischen Systemen

In diesem Kontext fokussieren neueste Forschungsartikel insbesondere auf die oben beschriebene Herausforderung, eine sinnvolle Zusammenführung und Kol- 
laboration von menschlichen und nicht-menschlichen Akteuren zu realisieren, also "best of both worlds" systematisch zu kombinieren (Böhmann et al. 2018). Grundhypothese hierbei ist, dass die heutigen und zukünftigen soziotechnischen Systeme stärker automatisiert und interaktiv, aber auch durch Offenheit und Durchlässigkeit - insbesondere bezogen auf Organisationsgrenzen - sowie (selbst-)lernenden Charakter gekennzeichnet sind.

\section{Fazit: Das Engineering von soziotechnischen Systemen als Gestaltungsauftrag}

Der vorliegende Beitrag zeigt zum einen die Grundlagen von Cloud, Hybrid Intelligence und digitaler Arbeit auf. Zum anderen setzt er diese ins Verhältnis zueinander und macht ihre Zusammenhänge deutlich. Aus einer Wirtschaftsinformatik-Perspektive heraus beschreiben wir, wie das Engineering - also die systematische Gestaltung unter Verwendung geeigneter Methoden, Modelle und Werkzeuge - von soziotechnischen Systemen sowie innovativen Dienstleistungen und entsprechenden Geschäftsmodellen unter Einbezug der zuvor vorgestellten disruptiven Entwicklungen und (technologischen) Trends erfolgen kann.

Das Einnehmen einer soziotechnischen Systemperspektive und die interdisziplinäre Zusammenarbeit stellen in der Folge Grundpfeiler dar, um die Potenziale zu heben, die neue Technologien wie Cloud, KI und HI sowie neue Formen digitaler Arbeit mit sich bringen. Ein Gestaltungsauftrag!

\section{Literatur}

Benlian, A.; Hess, T.; Buxmann, P. (2009): Drivers of SaaS-Adoption - An Empirical Study of Different Application Types', in: Business \& Information Systems Engineering, 1(5), S. 357. doi: 10.1007/s12599-009-0068-x

Benlian, A.; Kettinger, W. J.; Sunyaev, A.; Winkler, T. J. (2018): Special Section: The Transformative Value of Cloud Computing: A Decoupling, Platformization, 
and Recombination Theoretical Framework', in: Journal of Management Information Systems, 35(3), S. 719-739. doi: 10.1080/07421222.2018.1481634 Böhmann, T.; Leimeister, J. M.; Möslein, K. (2014): Service Systems Engineering, in: Business \& Information Systems Engineering, 6(2), S. 73-79. doi: $10.1007 / s 12599-014-0314-8$

Böhmann, T.; Leimeister, J. M.; Möslein, K. (2018): The New Frontiers of Service Systems Engineering, in: Business \& Information Systems Engineering, 60(5), S. 373-375. doi: 10.1007/s12599-018-0553-1

Cusumano, M. (2010): Cloud computing and SaaS as new computing platforms, in: Communications of the ACM, 53(4), S. 27. doi: 10.1145/1721654.1721667 Dellermann, D. et al. (2019) 'Hybrid Intelligence', Business and Information Systems Engineering (BISE), Online First.

Die Bundesregierung (2010): KI als Markenzeichen für Deutschland. https://www.bundesregierung.de/breg-de/themen/digital-made-in-de/ki-alsmarkenzeichen-fuer-deutschland-1549732 (Zugriff: 17. Dezember 2018).

Die Bundesregierung (2018): Eckpunkte der Bundesregierung für eine Strategie Künstliche Intelligenz. 18. Juli. https://www.bmas.de/SharedDocs/Downloads/ $\mathrm{DE} /$ Thema-Arbeitsmarkt/eckpunkte-strategie-ki.pdf?_blob=publicationFile\&v $=2$ (Zugriff: 17. Dezember 2018).

Durward, D.; Blohm, I.; Leimeister, J. M. (2016): Crowd Work, in: Business \& Information Systems Engineering, 58(4), S.. 281-286. doi: 10.1007/s12599016-0438-0

Durward, D.; Simmert, B.; Peters, C.; Blohm, I.; Leimeister, J. M. (2019): How to Empower the Workforce: Analyzing Internal Crowd Work as a Neo-SocioTechnical System, in: Proceedings of the Annual Hawaii International Conference on System Sciences, Maui, Hawaii, USA.

Hahn, C. (2016): Digitalisierung der IT-Industrie mit Cloud Plattformen Implikationen für Entwickler und Anwender, in: HMD Praxis der Wirtschaftsinformatik, 53(5), S. 594-606. doi: 10.1365/s40702-016-0259-0 Kleinschmidt, S.; Peters, C. (2017): Fostering business model extensions for ICT-enabled human-centered service systems. 13. Internationale Tagung 
Wirtschaftsinformatik: "Towards Thought Leadership in Digital Transformation". Universität St. Gallen.

Leimeister, J. M. (2012): Dienstleistungsengineering und -management. Berlin, Heidelberg: Springer.

Mell, P.; Grance, T. (2010): The NIST definition of cloud computing, in: Communications of the ACM, 53(6), S. 50.

Mrass, V.; Peters, C. (2019): Managing Work Systems for Complex Work via Crowdworking Platforms: How to Orchestrate the Interplay of Crowds, in: Proceedings of the Annual Hawaii International Conference on System Sciences, Maui, Hawaii, USA.

Mrass, V.; Peters, C.; Leimeister, J. M. (2018a): Managing Complex Work Systems via Crowdworking Platforms: How Deutsche Bank Explores AI Trends and the Future of Banking with Jovoto, in: Proceedings of the Annual Hawaii International Conference on System Sciences, Waikoloa, HI, USA.

doi: $10.24251 /$ HICSS.2018.431

Mrass, V.; Peters, C.; Leimeister, J. M. (2018b): Managing Complex Work Systems Via Crowdworking Platforms: How Intel and Hyve Explore Future Technological Innovations, in: SSRN Electronic Journal. doi: 10.2139/ssrn.3140628 Peters, C. (2015): Modularization of services. Dissertation. Kassel University Press $\mathrm{GmbH}$.

Tiwana, A.; Konsynski, B.; Bush, A. A. (2010): Research Commentary - Platform Evolution: Coevolution of Platform Architecture, Governance, and Environmental Dynamics, in: Information Systems Research, 21(4), S. 675-687. doi: $10.1287 /$ isre. 1100.0323 